\title{
The association between the subjective memory complaints scale and depressive state and cognitive impairment: a factor analysis
}

This article was published in the following Dove Press journal:

Neuropsychiatric Disease and Treatment

23 November 2015

Number of times this article has been viewed

\author{
Tetsu Tomita' \\ Norio Yasui-Furukori' \\ Norio Sugawara ${ }^{1,2}$ \\ Ippei Takahashi ${ }^{3}$ \\ Kaori Sawada ${ }^{3}$ \\ Kazuhiko Nakamura' \\ 'Department of Neuropsychiatry, \\ Graduate School of Medicine, \\ Hirosaki University, Hirosaki, ${ }^{2}$ Aomori \\ Prefectural Center for Mental Health \\ and Welfare, Aomori, ${ }^{3}$ Department \\ of Social Medicine, Graduate School \\ of Medicine, Hirosaki University, \\ Hirosaki, Japan
}

Background: We aimed to discriminate individuals with depressive state from individuals with cognitive impairment among community-dwelling people using the subjective memory complaints (SMC) scale.

Methods: The study group consisted of 289 volunteers (over 60 years old; 104 males and 185 females). Participants' SMCs were assessed using the SMC scale. The Japanese version of the Center for Epidemiologic Studies for Depression scale and the Mini-Mental State Examination were administered. Participants whose Center for Epidemiologic Studies for Depression scores were 16 or higher were defined as the depressive group and participants whose Mini-Mental State Examination scores were less than 24 were defined as the cognitive impairment group. Exploratory factor analysis was performed to identify the factor structure of the items of the SMC scale. A multiple logistic regression analysis of the association between depressive state and cognitive impairment and the score of each factor was performed.

Results: In the final factor analysis model, six items of the SMC scale remained, and a two-factor structure was adequate. Factor 1 included the items 8, 9, and 10 about thought or the ability to think; thus, Factor 1 was defined as "thought disturbance factor". Factor 2 included the items 1, 2, and 4 about memory or forgetfulness; thus, Factor 2 was defined as "memory disturbance factor". In the multiple logistic regression analysis, Factor 1 was significantly associated with depressive state and Factor 2 was significantly associated with cognitive impairment.

Conclusion: For individuals with SMCs, we might be able to discriminate depressive state or depression from cognitive impairment or dementia through a detailed investigation using the SMC scale.

Keywords: geriatric psychiatry, affective disorders, primary care

\section{Introduction}

A recent study has investigated the concept of subjective memory complaints (SMC) and suggested that the understanding of these complaints is of great relevance. ${ }^{1}$ Some studies indicate that the presence of any type of SMC may reflect objective memory impairment or be predictive of future dementia. ${ }^{2-5}$ Amariglio et al investigated the detail subjective symptoms of SMCs and suggested that difficulties following a group conversation or navigating familiar streets may be associated with severe memory impairment. ${ }^{6}$ In a study that used the Memory Impairment Screen (MIS) to evaluate participants' memory function, Modrego and Gazulla suggested that the MIS score might predict the conversion to Alzheimer's disease. ${ }^{7}$ By contrast, Silva et al reported that the severity of SMCs did not predict the future conversion to dementia. ${ }^{8}$

According to recent studies, SMCs are associated with not only dementia or actual cognitive impairment but also depressive state or major depressive disorder (MDD).
Correspondence: Tetsu Tomita Department of Neuropsychiatry, Graduate School of Medicine, Hirosaki University, 5 Zaifu-sho, Hirosaki 036-8562, Japan

Tel $+81 \quad 172395066$

$\mathrm{Fax}+8 \mid 172395067$

Email ttomita1984@yahoo.co.jp
Neuropsychiatric Disease and Treatment 20I5:I I 2935-294 I

Dovepress

http://dx.doi.org/1 0.2147/NDT.S93539 (c) (1) (5) 2015 Tomita et al. This work is published by Dove Medical Press Limited, and licensed under Creative Commons Attribution - Non Commercial (unported, v3.0) License. The full terms of the License are available at http://creativecommons.org/licenses/lby-nc/3.0/. Non-commercial uses of the work are permitted without any further permission from Dove Medical Press Limited, provided the work is properly attributed. Permissions beyond the scope of the License are administered by Dove Medical Press Limited. Information on how to request permission may be found at: http://www.dovepress.com/permissions.php 
Fischer et al studied the presence of SMCs among participants with and without MDD and reported that the subgroup of patients with MDD had significantly more SMCs $;{ }^{9}$ however, there were no significant differences between the two groups with respect to objective neuropsychological assessments. The decline in the self-evaluation of patients with MDD or their inhibition of thought may cause these patients' SMCs. Similarly, Balash et al revealed an association between the presence of SMCs and subsyndromal depression in healthy elders. ${ }^{10}$ Minett et al reported that in elderly participants, the presence of SMC was significantly associated with a higher score on the Geriatric Depression Scale. ${ }^{11}$ By contrast, Mowla et al investigated the cognitive functioning of patients with MDD and revealed that there was no correlation between SMCs and objective memory performance. ${ }^{12}$ Therefore, the meaning of SMCs among patients with MDD in this study is questionable.

In some studies that investigated SMCs, the SMC scale was used to evaluate the severity of SMCs. The SMC scale was advocated by Schmand et al and the items of SMC scale were derived from the Cambridge Examination for Mental Disorders of the Elderly. ${ }^{13,14}$ SMC scale consists of 10 items: item 1 "Do you have any complaints concerning your memory?", item 2 "Do other people find you forgetful?", item 3 "Do you ever forget names of family members or friends?", item 4 "Do you often forget where things are left?", item 5 "Do you often use notes to avoid forgetting things?", item 6 "Do you ever have difficulties in finding particular words?", item 7 "Did you ever lose your way in your neighborhood?", item 8 "Do you think more slowly than you used to?", item 9 "Do your thoughts ever become confused?", and item 10 "Do you have concentration problems?" These items were associated with difficulties in memory tasks of daily life and its internal consistency was showed. ${ }^{2,14}$ Ginó et al investigated both of elderly and of young healthy people using SMC scale and showed frequency of SMC and scores and the differences the characteristics of reported SMC between elderly and young people. ${ }^{15}$ Pires et al suggested that item 3 of the SMC scale was important and contributed to the variance of the total SMC score in clinical settings. ${ }^{16}$ Schmand et al reported that SMCs evaluated by the SMC scale might predict future dementia by longitudinal study; ${ }^{2,14}$ however, Silva et al investigated SMCs using the SMC scale in a clinical setting and found that the severity of SMCs did not predict the future conversion to dementia. ${ }^{8}$ There is no report to study the Japanese population or patients using SMC scale.

Thus, the current study examines the association between depression and dementia and utilizes the SMC scale to evaluate the severity and quality of SMCs. We previously reported that the presence of SMCs influences depressive symptoms and cognitive functioning. ${ }^{17}$ In the present study, we investigated the association between the items of the SMC scale and depressive state and cognitive impairment in community-dwelling people in Japan and identified the items that predict depression and cognitive impairment.

\section{Methods}

\section{Participants}

The study group consisted of 289 volunteers (over 60 years old; 104 males and 185 females) who participated in the Iwaki Health Promotion Project in 2013. The participants were residents of Iwaki district, Hirosaki City, in northern Japan. Iwaki district is a stable community with a population of approximately 12,000. The age and occupational distributions of this population are representative of a Japanese countryside community. Data collection for the present study and the project were approved by the Ethics Committee of Hirosaki University School of Medicine, and all subjects provided written informed consent prior to participating in the project. Demographic data (age, sex, and years of education) and lifestyle factors (smoking and drinking) were obtained from self-questionnaires and interviews.

\section{Assessment of SMCs}

Participants' SMCs were assessed using the SMC scale. The SMC scale consists of 10 items and its total score ranges from 0 to 21 . The score range of the items varied: items 6 and 7 were scored on 0 or 1 ; items $2,5,8,9$, and 10 were scored on $0-2$; and items 1,3 , and 4 were scored on $0-3$.

\section{Assessment of depressive state and cognitive impairment}

The Japanese version of the Center for Epidemiologic Studies for Depression (CES-D) scale was administered to all participants to measure their depressive status. ${ }^{18}$ The questionnaire has been widely used to measure depressive symptoms in community populations, and it is also used as a screening tool for depression. ${ }^{19}$ The CES-D is a 20 -item, self-report scale that focuses on depressive symptoms within the week prior to the administration of the questionnaire. The maximum score is 60 , and higher scores are associated with depression. CES-D scores of 16 or higher have generally been thought to indicate clinically relevant depressive symptoms, including both minor or subthreshold depression and MDD. ${ }^{20,21}$ In the present study, the CES-D score of 16 or higher was defined as depressive state and the participants 
whose CES-D scores 16 or higher were defined as the depressive group.

The Mini-Mental State Examination (MMSE) was given to all participants to measure their global cognitive status. This test assesses orientation to place and time, short-term memory, episodic long-term memory, subtraction, ability to construct a sentence, and oral language ability. The maximum score is 30 , and poor cognition is defined as a score less than $24 .^{22}$ In the present study, the MMSE score less than 24 was defined as cognitive impairment state and the participants with an MMSE score less than 24 were defined as the cognitive impairment group.

\section{Statistical analysis}

Descriptive statistical analyses were performed to describe the demographic and clinical variables. To compare the characteristics of the groups according to sex, depressive state, and cognitive impairment, an unpaired Student's $t$-test or Fisher's exact test was used to analyze variables.

Exploratory factor analysis was performed to identify the factor structure of the items of the SMC scale. We used Bartlett's test of sphericity to confirm the sufficient variability of the data for factor analysis. Estimation of the factors was performed by factoring the Pearson's correlation matrix by the maximum likelihood method with an oblique Promax rotation. Factor loadings with a minimum value in the range of \pm 0.40 were defined as building criteria for the exploratory factor analysis model. The items that did not reach a factor loading of \pm 0.40 were deleted from the model, and the remaining items were reanalyzed. The number of factors was determined by a scree plot and the Kaiser-Guttman rule; we adapted the factors with eigenvalue $>1$ for the model.

A direct multiple logistic regression analysis of the association between depressive state and cognitive impairment and the score of each factor was performed. In addition to that, we used the models that included the variables that showed significance in the $t$-tests as the independent variables. We used Hosmer-Lemeshow (H-L) test to determine the model goodness-of-fit. The data were analyzed using SPSS version 22 for Windows (SPSS Japan Inc., Tokyo, Japan. A $P$-value of $<0.05$ was considered as statistically significant.

\section{Results}

\section{Demographic data and scores}

The means \pm SDs of age, years of education, CES-D score, and MMSE score for all participants were 68.4 \pm 6.5 , $10.8 \pm 2.0,9.0 \pm 7.1$, and $28.4 \pm 2.0$, respectively. The participants consisted of 104 male (36.0\%) and 185 female
(64.0\%) participants. Forty-six participants (15.9\%) showed depressive state and ten participants $(3.5 \%)$ showed cognitive impairment. Table 1 shows the demographic data, the results of the depressive state and cognitive impairment assessments, and the scores of the items of the SMC scale.

There were no significant differences between male and female participants in terms of age and years of education. There were no significant differences between male and female participants in CES-D score and the prevalence rate of the depressive state, but the MMSE score and the prevalence rate of cognitive impairment were higher in male participants than in female participants. There were significant differences between the participants with and without depressive state in terms of age, MMSE score and the rate of the participants with cognitive impairment; the participants with depressive state were older and showed lower MMSE scores and higher cognitive impairment rates. There were significant differences between the participants with and without cognitive impairment in terms of age and sex; the participants with cognitive impairment were older and consisted of more male participants.

The score of item 5 of the SMC scale was significantly higher in female participants than in male participants. When comparing the participants with and without depressive state, the total score of the SMC scale and items 1, 3, 4, 6, 8, 9, and 10 were significantly higher in the participants with depressive state than in the participants without depressive state. When comparing the participants with and without cognitive impairment, item 2 was significantly higher in the participants with cognitive impairment than in the participants without cognitive impairment.

\section{Factor analysis}

Table 2 shows the factor loadings of the SMC scale. According to the results of oblique Promax rotation, some items of the SMC scale were excluded according to the criteria shown above. In the final model, six items of the SMC scale remained. The Kaiser-Meyer-Olkin measure for sample adequacy was 0.797 , and Bartlett's test of sphericity was $P<0.001$. Figure 1 shows the scree plot. According to the Kaiser-Guttman rule and scree plot, a two-factor structure was adequate.

Factor 1 included the items about thought or the ability to think; thus, Factor 1 was defined as "thought disturbance factor". Factor 2 included the items about memory or forgetfulness; thus, Factor 2 was defined as "memory disturbance factor". The correlation coefficient between Factors 1 and 2 was 0.587 . 


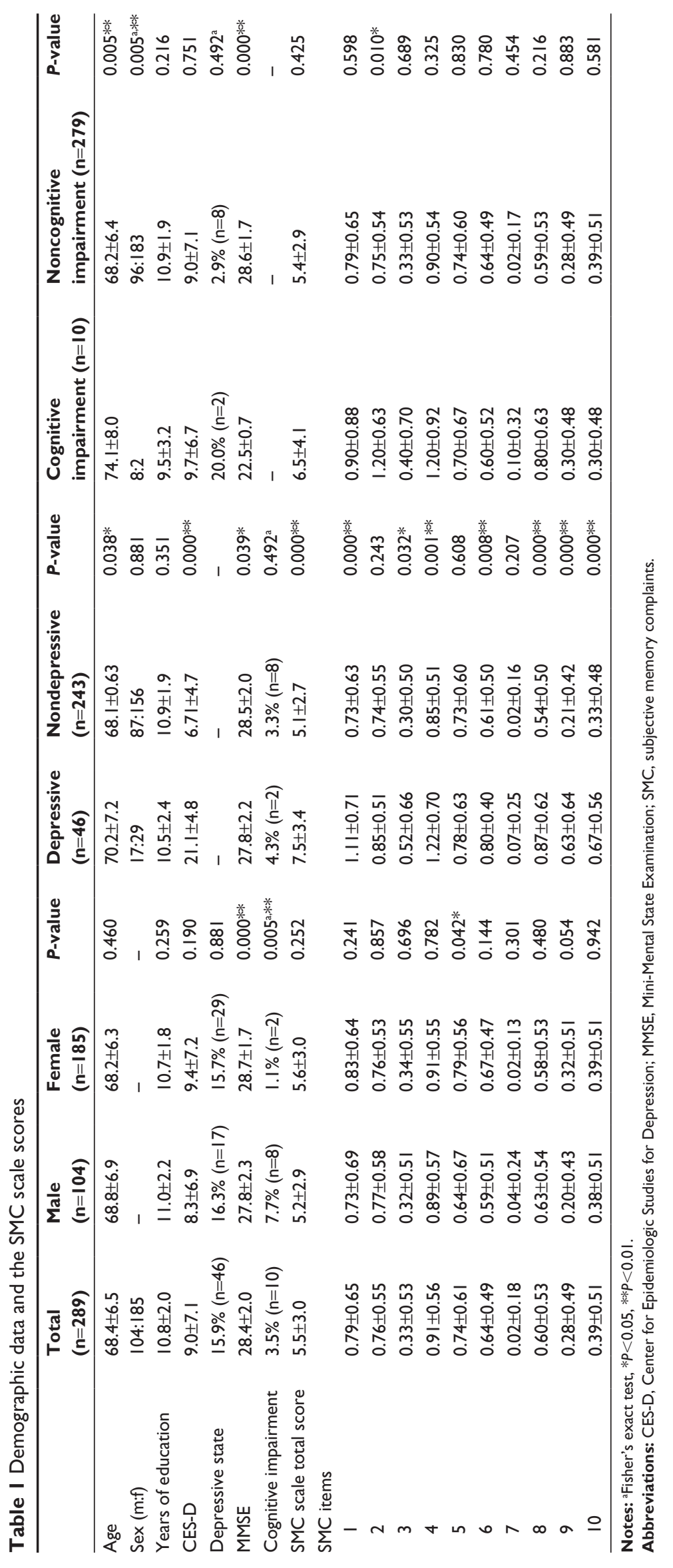


Table 2 Factor loadings of the SMC scale items

\begin{tabular}{llll}
\hline Questions & & Factor I & Factor 2 \\
\hline 10 & Do you have concentration problems? & 0.918 & -0.134 \\
9 & Do your thoughts ever become confused? & 0.584 & 0.075 \\
8 & Do you think more slowly than you used to? & 0.535 & 0.228 \\
2 & Do other people find you forgetful? & -0.098 & 0.711 \\
1 & Do you have any complaints concerning your memory? & 0.055 & 0.685 \\
4 & Do you often forget where things are left? & 0.113 & 0.562 \\
& Contribution rate & $48.2 \%$ & $17.2 \%$ \\
\hline
\end{tabular}

Abbreviation: SMC, subjective memory complaints.

\section{Multiple logistic regression analysis}

Table 3 shows the results of the multiple logistic regression analysis that used Factors 1 and 2 to predict depressive state and cognitive impairment.

Using depressive state as the dependent factor, Factor 1 was significantly associated with depressive state, but Factor 2 was not; model 1. Using cognitive impairment as the dependent factor, Factor 2 was significantly associated with cognitive impairment, but Factor 1 was not; model 2. Factors 1 and 2 showed different associations with depressive state and cognitive impairment; Factor 1 predicted depressive state, and Factor 2 predicted cognitive impairment. $P$-values of $\mathrm{H}-\mathrm{L}$ tests of models 1 and 2 were 0.870 and 0.055 , respectively.

According to the results of the $t$-tests shown above, age and sex were defined as the important variables, so these were included as the independent variables in further models of multiple logistic regression analysis. In model 3, which included age and sex as independent variables, Factor 1 was similarly and significantly associated with depressive state, but Factor 2 was not. In model 4, which included age and

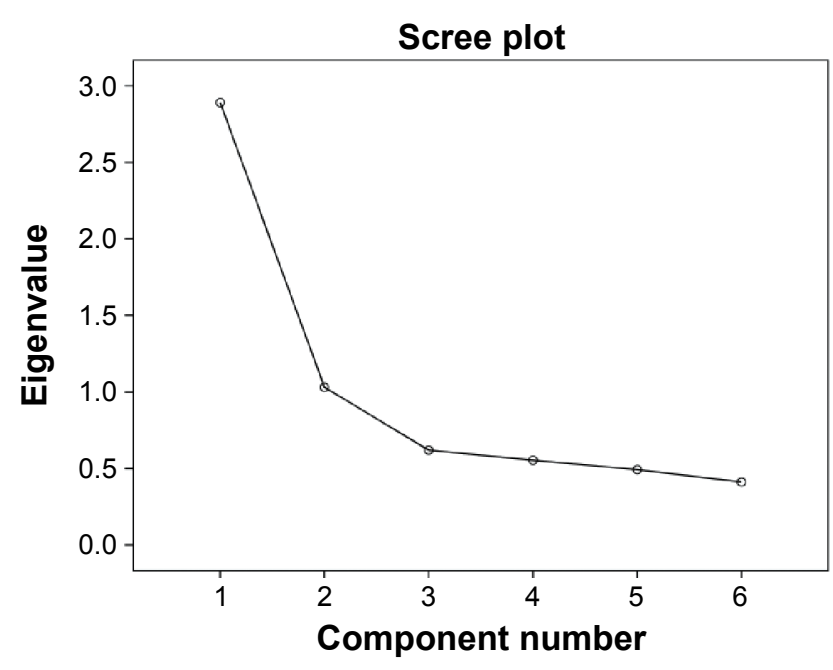

Figure I Scree plot of subjective memory complaints scale items. sex as independent variables, Factors 1 and 2 were not significantly associated with cognitive impairment, but age and sex showed a significant association. $P$-values of $\mathrm{H}-\mathrm{L}$ tests of models 1 and 2 were 0.825 and 651 , respectively.

\section{Discussion}

In the present study, we identified the SMC scale items and factors that are significantly associated with depressive state and cognitive impairment. This current report is the first to investigate the SMC items and discriminate the factors associated with depressive state and cognitive impairment. We might discriminate depressive state from cognitive impairment or screen for these diagnoses by administering the SMC scale and focusing on the items included in Factors 1 and 2 for patients with SMCs.

The SMC scale might have the potential to more easily discriminate depressive state from cognitive impairment than other tools, because the scale is a simple self-report questionnaire and participants might be able to complete it quickly. In a previous study, Monette and Leach investigated the discrimination of mild cognitive impairment and depression using the Kaplan-Baycrest Neurocognitive Assessment (KBNA). ${ }^{23}$ They revealed that the assessment of KBNA had the potential to distinguish participants with depression from participants with mild cognitive impairment among participants with SMCs. Whereas we investigated community-dwelling people in the present study, Monette and Leach investigated only participants with SMCs; thus, their study was more clinical. Furthermore, it takes approximately 50-65 minutes to assess the KBNA. ${ }^{23}$ A simpler and more clinical assessment tool is needed for the strict and useful discrimination of depression and dementia in clinical settings.

The items of the SMC scale refer to general subjective symptoms about memory, but in the present study, items 8 ("Do you think more slowly than you used to?"), 9 ("Do your thoughts ever become confused?"), and 10 ("Do you have concentration problems?") were significantly associated with depressive state according to the results of the multiple 
Table 3 Results of logistic regression analysis

\begin{tabular}{|c|c|c|c|c|c|c|c|}
\hline Model & Dependent variables & $\begin{array}{l}\text { Independent } \\
\text { variables }\end{array}$ & $\beta$ & $\begin{array}{l}\text { Standard } \\
\text { error }\end{array}$ & $\begin{array}{l}\text { Odds } \\
\text { ratio }\end{array}$ & $\begin{array}{l}95 \% \text { confidence } \\
\text { interval }\end{array}$ & $P$-value \\
\hline \multirow[t]{2}{*}{1} & Depressive state & Factor I & 0.584 & 0.156 & 1.793 & $1.320-2.434$ & $0.000 * *$ \\
\hline & & Factor 2 & 0.177 & 0.141 & 1.194 & $0.906-1.574$ & 0.208 \\
\hline \multirow[t]{2}{*}{2} & Cognitive impairment & Factor I & -0.211 & 0.286 & 0.810 & $0.463-1.418$ & $0.46 \mathrm{I}$ \\
\hline & & Factor 2 & 0.529 & 0.265 & 1.698 & $1.010-2.854$ & $0.046^{*}$ \\
\hline \multirow[t]{4}{*}{3} & Depressive state & Factor I & 0.567 & 0.158 & 1.763 & $1.295-2.40 \mid$ & $0.000 * *$ \\
\hline & & Factor 2 & 0.165 & 0.141 & 1.179 & $0.894-1.555$ & 0.243 \\
\hline & & Age & 0.024 & 0.026 & 1.024 & $0.974-1.077$ & 0.350 \\
\hline & & Sex & -0.136 & 0.355 & 0.873 & $0.435-1.752$ & 0.702 \\
\hline \multirow[t]{4}{*}{4} & Cognitive impairment & Factor I & -0.224 & 0.311 & 0.799 & $0.434-1.47 \mid$ & 0.472 \\
\hline & & Factor 2 & 0.409 & 0.264 & 1.505 & $0.898-2.523$ & 0.121 \\
\hline & & Age & 0.102 & 0.048 & 1.107 & $1.009-1.216$ & $0.032^{*}$ \\
\hline & & Sex & -1.947 & 0.818 & 0.143 & $0.029-0.709$ & $0.017^{*}$ \\
\hline
\end{tabular}

Notes: $* P<0.05, * * P<0.01$.

logistic regression analysis. The symptoms that are presented in these items are frequent and common in depression and are included in the diagnostic criteria of major depressive episode in the Diagnostic and Statistical Manual of Mental Disorders-5; thus, it is easy to understand why these items (Factor 1) are associated with depressive state.

The meaning of discussing the association between depressive state and SMCs was questionable because the results of previous reports on the association were inconsistent. ${ }^{9-12}$ Typically, assessments of SMCs were very general and included symptoms other than memory symptoms or symptoms associated with only dementia. We might be able to show consistent results concerning the association between depression and SMCs by focusing on depression or depressive symptoms. According to the results of the present study, we should pay attention to depressive symptoms other than SMCs when we evaluate the neuropsychiatric symptoms of patients with SMCs using a scale.

\section{Limitations}

There were some limitations of the present study. First, we only investigated community-dwelling people. We believe that the SMC scale is useful for screening depressive state and cognitive impairment in individuals with SMCs, but we do not know whether the results of the present study are generalizable to patients visiting clinical settings due to their SMCs. Further investigation is needed to apply the SMC scale in clinical settings. Second, in the present study, we did not investigate cutoff values to distinguish between depressive state and cognitive impairment. The critical cutoff values are more useful in clinical settings than in determining the association between some items of the SMC scale and depressive state and cognitive impairment. Third, the results lack information about sex-specific effects on the association between factors or items of the SMC scale and depressive state and cognitive impairment. Many previous studies reported sex differences in the prevalence and characteristics of depressive state and cognitive impairment, and we previously reported sex differences in the association between the presence of SMCs and depressive symptoms and cognitive function and in the prediction of the effectiveness of treatment for patients with depression. ${ }^{17,24}$ The investigation of the effects of sex might increase the importance of the present results. Fourth, the reliability of discriminating depressive state from cognitive impairment by Factors 1 and 2 is limited because the correlation coefficient between two factors was 0.587 , and Factors 1 and 2 did not show significant associations with cognitive impairment. Age and sex might be more important in predicting cognitive impairment than Factors 1 and 2. Further investigation is required to define more reliable factors or values to discriminate these two statements.

\section{Conclusion}

We investigated the association between the items of the SMC scale and depressive state and cognitive impairment and identified the specific items and factors that were associated with depressive state and cognitive impairment. For individuals with SMCs, we might be able to discriminate depressive state or depression from cognitive impairment or dementia through a detailed investigation using the SMC scale.

\section{Acknowledgments}

This study was funded by a Grant-in-Aid for Scientific Research (KAKENHI) from the Japan Society for the Promotion of Research (JSPS, 15K19239, 15K19710, and 
15H04754) and a grant from the Hirosaki Research Institute for Neurosciences. The authors would like to thank all of their coworkers on this study for their skillful contributions to collecting and managing the data.

\section{Author contributions}

NY-F and KN designed the study and wrote protocol. TT managed the literature searches and analysis. NS managed analysis. IT and KS collected the data. All authors contributed toward data analysis, drafting and revising the paper and agree to be accountable for all aspects of the work.

\section{Disclosure}

NY-F has received grant/research support or honoraria from, and been on the speakers of Asteras, Dainippon, Eli Lilly, GSK, Janssen-Pharma, Meiji, Mochida, MSD, Otsuka, Pfizer, Takada, and Yoshitomi. The funders had no role in study design, data collection and analysis, decision to publish, or preparation of the manuscript. The other authors report no conflicts of interest in this work.

\section{References}

1. Roberts JL, Clare L, Woods RT. Subjective memory complaints and awareness of memory functioning in mild cognitive impairment: a systematic review. Dement Geriatr Cogn Disord. 2009;28:95-109.

2. Schmand B, Jonker C, Geerlings MI, Lindeboom J. Subjective memory complaints in the elderly: depressive symptoms and future dementia. Br J Psychiatry. 1997;171:373-376.

3. Clarnette RM, Almeida OP, Forstl H, Paton A, Martins RN. Clinical characteristics of individuals with subjective memory loss in Western Australia: results from a cross-sectional survey. Int J Geriatr Psychiatry. 2001;16:168-174.

4. Benito-Leon J, Mitchell AJ, Vega S, Bermejo-Pareja F. A populationbased study of cognitive function in older people with subjective memory complaints. J Alzheimers Dis. 2010;22:159-170.

5. Genziani M, Stewart R, Bejot Y, Amieva H, Artero S, Ritchie K. Subjective memory impairment, objective cognitive functioning and social activity in French older people: findings from the Three Cities study. Geriatr Gerontol Int. 2013;13:139-145.

6. Amariglio RE, Townsend MK, Grodstein F, Sperling RA, Rentz DM. Specific subjective memory complaints in older persons may indicate poor cognitive function. J Am Geriatr Soc. 2011;59:1612-1617.

7. Modrego PJ, Gazulla J. The predictive value of the memory impairment screen in patients with subjective memory complaints: a prospective study. Prim Care Companion CNS Disord. 2013;15:pii:PCC.12m01435.
8. Silva D, Guerreiro M, Faria C, Maroco J, Schmand BA, Mendonca A. Significance of subjective memory complaints in the clinical setting. $J$ Geriatr Psychiatry Neurol. 2014;27:259-265.

9. Fischer C, Schweizer TA, Atkins JH, et al. Neurocognitive profiles in older adults with and without major depression. Int J Geriatr Psychiatry. 2008;23:851-856.

10. Balash Y, Mordechovich M, Shabtai H, Giladi N, Gurevich T, Korczyn AD. Subjective memory complaints in elders: depression, anxiety, or cognitive decline? Acta Neurol Scand. 2013;127:344-350.

11. Minett TS, Da Silva RV, Ortiz KZ, Bertolucci PH. Subjective memory complaints in an elderly sample: a cross-sectional study. Int $J$ Geriatr Psychiatry. 2008;23:49-54.

12. Mowla A, Ashkani H, Ghanizadeh A, Dehbozorgi GR, Sabayan B, Chohedri AH. Do memory complaints represent impaired memory performance in patients with major depressive disorder? Depress Anxiety. 2008;25:E92-E96.

13. Roth M, Tym E, Mountjoy CQ, Huppert FA. Camdex: The Cambridge Examination for Mental Disorders of the Elderly. Cambridge, Cambridgeshire: Cambridge University Press; 1988.

14. Schmand B, Jonker C, Hooijer C, Lindeboom J. Subjective memory complaints may announce dementia. Neurology. 1996;46:121-125.

15. Ginó S, Mendes T, Maroco J, et al. Memory complaints are frequent but qualitatively different in young and elderly healthy people. Gerontology. 2010;56:272-277.

16. Pires C, Silva D, Maroco J, et al. Memory complaints associated with seeking clinical care. Int J Alzheimers Dis. 2012;2012:725329.

17. Tomita T, Norio YF, Sato Y, et al. Sex differences in the prediction of the effectiveness of paroxetine for patients with major depressive disorder identified using a receiver operating characteristic curve analysis for early response. Neuropsychiatr Dis Treat. 2014;10:599-606.

18. Shima S, Shikano T, Kitamura T. New self-rating scales for depression. Clin Psychiatry. 1985;27:717-723 (Japanese).

19. Zich JM, Attkisson CC, Greenfield TK. Screening for depression in primary care clinics: the CES-D and the BDI. Int J Psychiatry Med. 1990;20:259-277.

20. Berkman LF, Berkman CS, Kasl S, et al. Depressive symptoms in relation to physical health and functioning in the elderly. Am J Epidemiol. 1986;124:372-388.

21. Beekman AT, Deeg DJ, Van Limbeek J, Braam AW, De Vries MZ, Van Tilburg W. Criterion validity of the Center for Epidemiologic Studies Depression scale (CES-D): results from a community-based sample of older subjects in The Netherlands. Psychol Med. 1997;27:231-235.

22. Folstein MF, Folstein SE, McHugh PR. "Mini-mental state". A practical method for grading the cognitive state of patients for the clinician. J Psychiatr Res. 1975;12:189-198.

23. Monette MC, Leach L. Discrimination of the cognitive profiles of MCI and depression using the KBNA. Can J Neurol Sci. 2013;40: 670-677.

24. Tomita T, Sugawara N, Kaneda A, et al. Sex-specific effects of subjective memory complaints with respect to cognitive impairment or depressive symptoms. Psychiatry Clin Neurosci. 2014;68:176-181.
Neuropsychiatric Disease and Treatment

\section{Publish your work in this journal}

Neuropsychiatric Disease and Treatment is an international, peerreviewed journal of clinical therapeutics and pharmacology focusing on concise rapid reporting of clinical or pre-clinical studies on a range of neuropsychiatric and neurological disorders. This journal is indexed on PubMed Central, the 'PsycINFO' database and CAS,

\section{Dovepress}

and is the official journal of The International Neuropsychiatric Association (INA). The manuscript management system is completely online and includes a very quick and fair peer-review system, which is all easy to use. Visit http://www.dovepress.com/testimonials.php to read real quotes from published authors. 